\title{
Characteristics of discrete VLF falling-tone chorus emissions observed at low latitude ground station Jammu
}

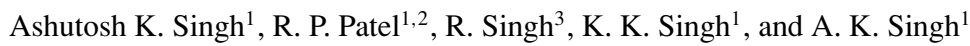 \\ ${ }^{1}$ Atmospheric Research Lab., Department of Physics, Banaras Hindu University, Varanasi-221005, India \\ ${ }^{2}$ Department of Physics, M.M.H. P.G. College, Ghaziabad, India \\ ${ }^{3}$ Indian Institute of Geomagnetism, Navi Mumbai, India
}

(Received May 25, 2009; Revised June 24, 2009; Accepted June 25, 2009; Online published November 30, 2009)

\begin{abstract}
The discrete VLF chorus emissions recorded at low latitude ground station Jammu (geomag. lat. $=22^{\circ} 26^{\prime} \mathrm{N}$, $L=1.17$ ) are reported and their characteristics based on spectral analysis have been carried out. These discrete chorus emissions are falling tone emissions which are rarely observed at low latitude ground stations. These discrete chorus emissions are generated during a strong geomagnetic storm period of 2-7 May, 1998. We have computed the frequency sweep rate, repetition period and source region of the individual chorus elements. It is observed that the sweep rate increases with time.
\end{abstract}

Key words: VLF emissions, whistler-mode waves, geomagnetic storm.

\section{Introduction}

Chorus emissions are one of the most intriguing signals among natural ELF/VLF radiation. These emissions usually consist of a succession of discrete elements with rising (falling) frequency having repetition period of $T \sim 0.1-1 \mathrm{~s}$ and typical duration of chorus events is $0.5-1 \mathrm{~h}$ (Helliwell, 1965). The chorus emissions are generally generated in the near equatorial region by the cyclotron instability of radiation belt electrons (Helliwell, 1967; Sazhin and Hayakawa, 1992). Chorus are characterized by a sequence of discrete elements depending upon their spectrum as intense tones, short duration, generally rising and often overlapping and occurring in association with disturbed magnetospheric conditions (Helliwell, 1965; Sazhin and Hayakawa, 1992). Although it is generally accepted that the generation mechanism of these emissions is connected with the cyclotron instability of whistler-mode waves and radiation belt electrons (Helliwell, 1967), the generation mechanism of these emissions and formation of spectrum of separate elements are still a subject of active experimental and theoretical research (Smith et al., 1996; Trakhtengerts, 1999; Singh et al., 2000; Lauben et al., 2002; Santolik and Gurnett, 2003; Titova et al., 2003; Singh and Singh, 2004; Singh and Ronnmark, 2004; Santolik, 2008; Bortnik et al., 2008; Omura et al., 2008 and references there in).

The generation mechanism of chorus has been extensively studied in the past (Sazhin and Hayakawa, 1992 and references therein). The non-linear cyclotron resonance interaction between whistler mode waves and counter streaming electrons is most widely used theory (Trakhtengerts, 1999; Singh et al., 2000; Titova et al., 2003; Singh and Patel, 2004). During the development of cyclotron insta-

Copyright (c) The Society of Geomagnetism and Earth, Planetary and Space Sciences (SGEPSS); The Seismological Society of Japan; The Volcanological Society of Japan; The Geodetic Society of Japan; The Japanese Society for Planetary Sciences; TERRAPUB. bility, a singularity in the form of the step on the distribution function is formed which serves as a boundary in velocity space between resonant and non-resonant electrons. When the resonant region is small, the phase effects become important and backward wave oscillator (BWO) regime is realized and discrete emissions like chorus is generated. Singh and Patel (2004) using BWO mechanism tried to explain some features of chorus observed at Indian Antarctica Station, Maitri. The observation of chorus emissions at low latitude ground station Jammu are unusual in the sense that most of reported chorus emissions observed from either satellite (Cornilleau-Wehrlin et al., 1978; Hattori et al., 1991; Santolik and Gurnett, 2003) or from high latitude stations. The falling tone emissions were rarely reported at any low latitude ground stations.

In the present paper, we present a detailed spectral analysis of the discrete VLF falling-tone chorus emissions recorded at low latitude ground station Jammu (geomag. lat. $=22^{\circ} 26^{\prime} \mathrm{N} ; L=1.17$ ) during the routine recording of whistlers from January 1998 to December 1998. Section 2 describes the experimental setup and data analysis. The complete spectral analysis of the falling tones has been explained in Section 3. The results are discussed in Section 4 and finally, Section 5 gives the conclusions of the study.

\section{Experimental Setup and Data}

At low latitude ground station Jammu ( $L=1.17)$, the broad band discrete chorus emissions were received by a Ttype antenna, pre- and main-amplifiers and tape recorder having band width of $50 \mathrm{~Hz}-15 \mathrm{kHz}$. T-type antenna is 25 meter in vertical length and 6 meter long horizontally and $3.2 \mathrm{~mm}$ in diameter. The impedance is about $1 \mathrm{M} \Omega$. The antenna is rendered aperiodic with the help of a suitable RC network, to avoid any possible ringing effect. This is erected at a suitable distance from the main building to reduce the power line hum and any other type of man made 


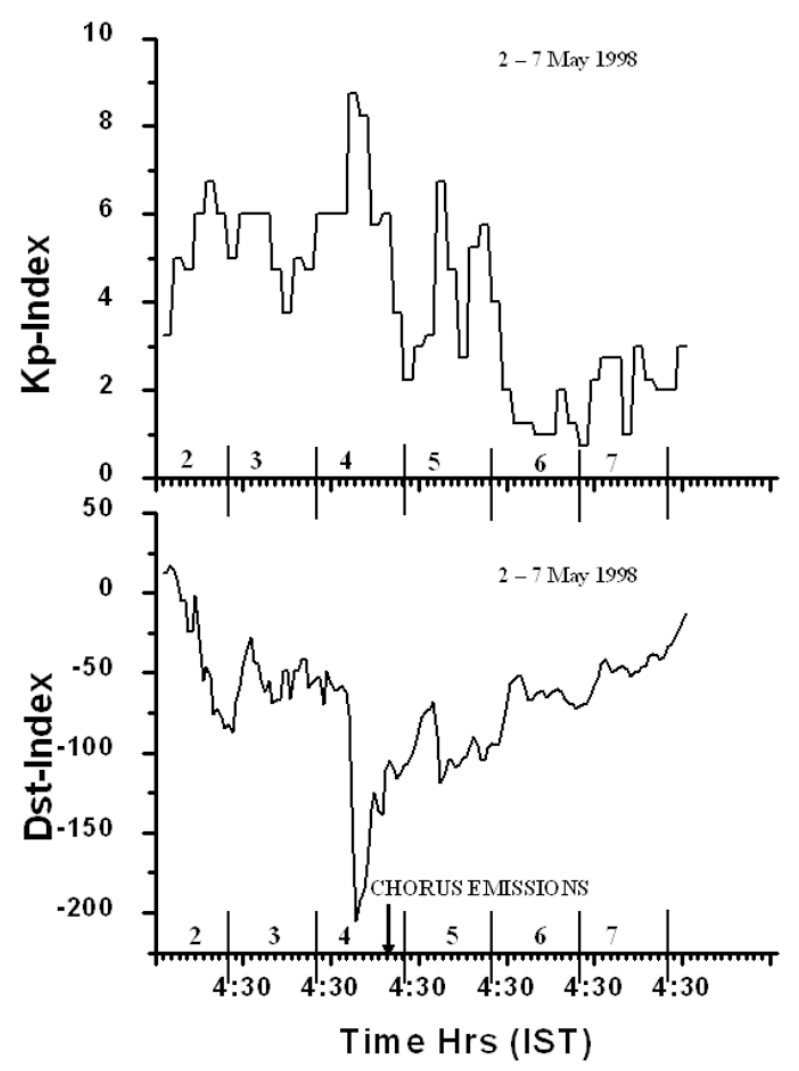

Fig. 1. The variation of $K_{\mathrm{p}}$-index and $D_{\mathrm{st}}$-index on geomagnetic storm period of 2-7 May 1998. The occurrence of chorus emissions is also marked by arrow.

noises. Between the antenna and pre/main amplifier, an active filter unit is introduced to reduce the local noise to a minimum in the frequency range $100 \mathrm{~Hz}$ to $500 \mathrm{~Hz}$. The filter is constructed from a simple RC network along with operational amplifier to be operated in negative feedback mode. The lower cutoff frequency of the filter is about $600 \mathrm{~Hz}$. The gain of the pre/main amplifier is varied from 0 to $40 \mathrm{~dB}$ to avoid overloading of the amplifier at the time of great VLF activity. The observations were taken continuously both during day and night times. The VLF data were stored on the magnetic tapes, which were analyzed using 'Raven' software. This software is developed under Cornell Lab. of Ornithology bioacoustics research program, USA for the acquisition, visualization measurement and analysis of sounds.

In this paper, the VLF falling-tone discrete chorus emissions recorded in large numbers during the night hours of 4-5 May 1998 is analyzed. These emissions were recorded during strong geomagnetic period of 2-7 May 1998 with minimum of $D_{\text {st }}$ Index $-204 \mathrm{nT}$ as shown in Fig. 1. The emissions were recorded in recovery phase of geomagnetic storm period. The most intense discrete chorus emissions were started during the night of 4-5 May, when the magnetic activity was highest $\left(\Sigma K_{P}=43\right)$ during the recovery phase of storm.

\section{Spectral Analysis}

Typical frequency-time spectrogram of discrete VLF falling-tone chorus emissions recorded during nighttime at 2250-2330 hrs (IST), on 4-5 May 1998 at Jammu are shown in Fig. 2. The color in this figure represents an estimate of the logarithm of the relative power in decibel $(\mathrm{dB})$ in a particular band over particular time interval. Discrete VLF chorus emissions (fallers) in sufficient numbers in the frequency range $2.5 \mathrm{KHz}$ to $5.5 \mathrm{KHz}$ were observed for about 1 hour. The repetition period of these events are not equal everywhere and increases with time. Figure 2 shows the VLF falling-tone chorus emissions observed at Jammu in four different stages of time. Figure 2(a) shows the chorus emissions in the beginning of the observations at 2250 hrs (IST). Figure 2(b) shows chorus emissions at 23:00 hrs IST with the increased repetition period. Figure 2(c) shows the chorus emissions at 23:20 hrs IST and Fig. 2(d) shows discrete chorus emissions at the end of observations.

About twenty five discrete VLF emissions, which are falling tones observed at $2250 \mathrm{hrs}$ IST are shown by Fig. 2(a). The observed discrete emissions have the following parameters: $f_{\min }=3.1 \mathrm{kHz}, f_{\max }=4.4 \mathrm{kHz}$, average upper band frequency $f_{\mathrm{UB}}=4.4 \mathrm{kHz}$, average frequency sweep rate $d f / d t=-11.01 \mathrm{kHz} / \mathrm{sec}$ and average duration of each discrete emissions $T=0.15 \mathrm{sec}$. Figure 2(b) shows about fifteen discrete chorus emissions observed at $2300 \mathrm{hrs}$ IST in the frequency range of about $3.0-5.3 \mathrm{kHz}$. In this case the observed discrete VLF chorus emissions have the following parameters: $f_{\min }=3.4 \mathrm{kHz}, f_{\max }=4.9 \mathrm{kHz}$, average $f_{\mathrm{UB}}=4.9 \mathrm{kHz}$, average frequency sweep rate $d f / d t=-7.5 \mathrm{kHz} / \mathrm{sec}$ and average duration of each discrete emissions $T=0.23 \mathrm{sec}$. In third case which is shown in Fig. 2(c), we have shown frequency-time spectrogram of chorus emissions at $2320 \mathrm{hrs}$ IST in the frequency range of about $3.0-5.3 \mathrm{kHz}$. The observed discrete emissions have the following parameters: $f_{\min }=3.4 \mathrm{kHz}, f_{\max }=4.7 \mathrm{kHz}$, average $f_{\mathrm{UB}}=4.7 \mathrm{kHz}$, average frequency sweep rate $d f / d t=-4.3 \mathrm{kHz} / \mathrm{sec}$ and average duration of each discrete emissions $T=0.43 \mathrm{sec}$. In the end of the observation at 2327 hrs IST, Fig. 2(d) shows the chorus emissions which are in the frequency range of $3.5-6.2 \mathrm{kHz}$. The observed discrete emissions for the set have the following parameters: $f_{\text {min }}=4.8 \mathrm{kHz}, f_{\text {max }}=5.4 \mathrm{kHz}$, average $f_{\mathrm{UB}}=5.4 \mathrm{kHz}$, average frequency sweep rate $d f / d t=-0.86 \mathrm{kHz} / \mathrm{sec}$ and average duration of each discrete emissions $T=1.10 \mathrm{sec}$. All the above parameters of the discrete chorus emissions (falling tones) are tabulated in Table 1.

\section{Discussions}

The dynamic spectrum of discrete VLF chorus emissions observed at low latitude ground station Jammu shows that the occurrence rate of these emissions is low and sporadic. It is also seen that the number of discrete chorus emissions observed during strong magnetic storm period are large. Similar occurrence rate of VLF emissions recorded at Gulmarg were reported by Singh et al. (2000). Recently, Singh et al. (2007) have presented a large number of VLF emissions generated during the strong magnetic disturbances in recovery phase of storm. The direction finding measure- 

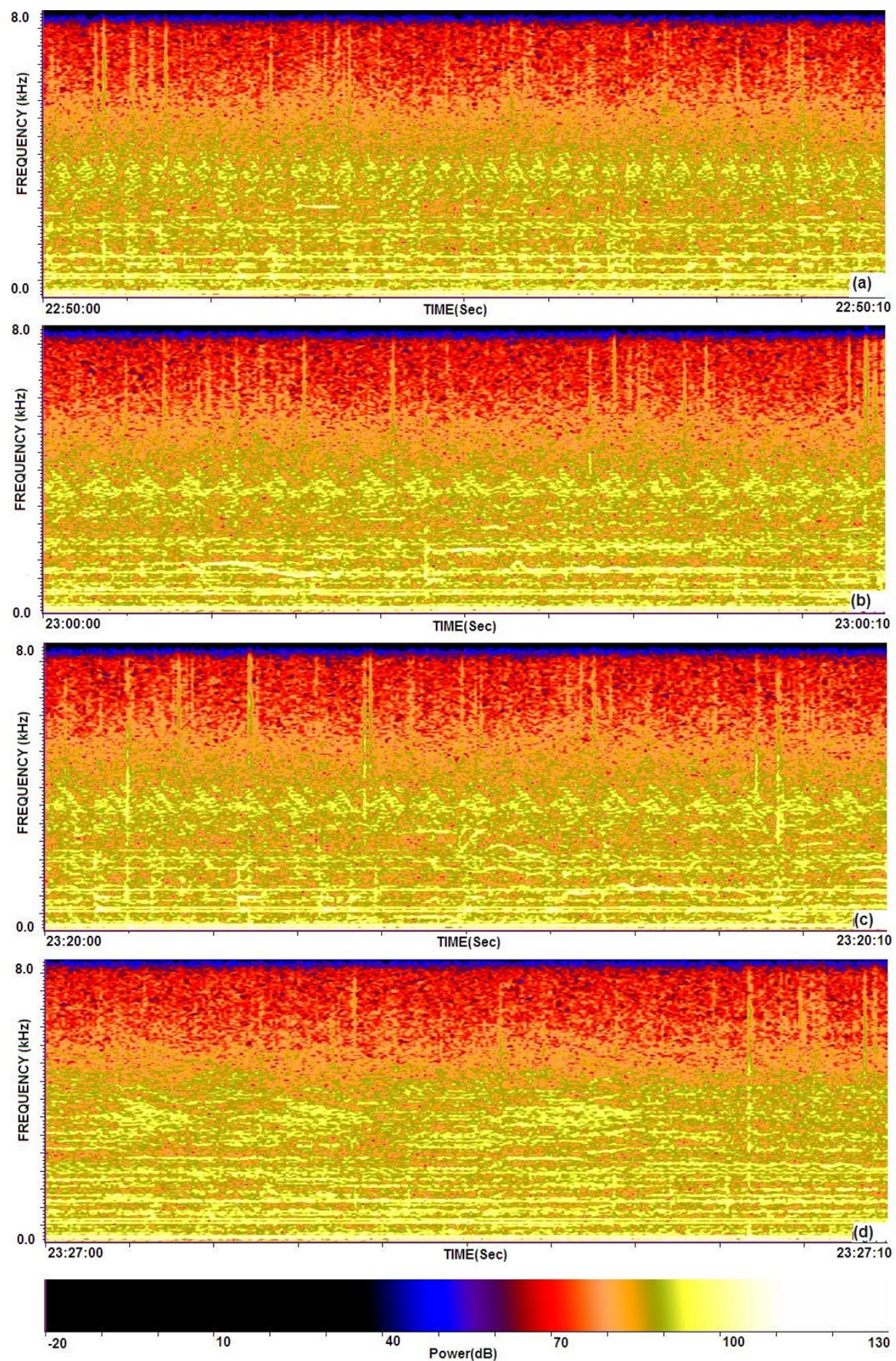

Fig. 2. Examples of VLF discrete chorus emissions recorded at Jammu on 4th May 1998 at 22:50 to 23:30 hrs IST.

ment of VLF and ELF hiss triggered chorus emissions were analyzed by Hattori and Hayakawa (1994) and found that the azimuth angle values for both of them are the same. The most frequent observations of chorus events by satellites near the geomagnetic equator (Tsurutani and Smith, 1974; Burtis and Helliwell, 1976) support the idea that the source of chorus emissions are mostly localized near equatorial region.
The generation of chorus emissions is derived by the injection of substorm electrons (Tsurutani and Smith, 1974) that interact with whistler mode waves through the cyclotron resonance (Andronov and Trakhtengerts, 1964; Kennel and Petschek, 1966). By analyzing POLAR satellite data Lauben et al. (2002) have studied various source characteristics of chorus emissions and indicated the source region near the magnetic equator. The range of observed 
Table 1. Observed parameters of discrete VLF falling-tone chorus emissions recorded at low latitude ground station Jammu.

\begin{tabular}{clrcc}
\hline S. No. & Parameter & Set I & Set II & Set III \\
\hline 1. & $L_{\text {Source }}$ & 4.62 & 4.5 & 4.56 \\
2. & $f_{\text {UB }}(\mathrm{kHz})$ & 4.45 & 4.85 & 4.66 \\
3. & $f_{\mathrm{LC}}(\mathrm{kHz})$ & 2.98 & 3.33 & 3.43 \\
4. & $T(\mathrm{sec})$ & 0.15 & 0.31 & 0.43 \\
5. & $d f / d t(\mathrm{kHz} / \mathrm{sec})$ & -11.01 & -7.49 & -4.25 \\
\hline
\end{tabular}

chorus frequencies is controlled by the equatorial electron gyrofrequencies (Burtis and Helliwell, 1976).

The maximum of chorus intensity is found to move towards lower latitudes with the increase in the geomagnetic activity, which may be due to the motion of the propagating channels and source region towards the Earth (Smirnova et al., 1976; Smirnova, 1984). We have followed the Upper Boundary Frequency (UBF) method developed by Smirnova (1984), to find out the location of source for the recorded discrete VLF chorus emissions. The upper boundary frequency of the ground based observation of discrete chorus events is determined on the assumption of dipolar geomagnetic field configuration, by the half equatorial electron gyrofrequency in the generation region, irrespective of the latitude of the observation station. According to Smirnova (1984), the $L$-value of the observed discrete VLF chorus emissions source is written as

$$
L=\left(440 / f_{\mathrm{UB}}\right)^{1 / 3}
$$

where $f_{\mathrm{UB}}$ is the upper cut-off boundary frequency of the observed discrete chorus events in $\mathrm{kHz}$. Using Eq. (1), the $L$-value of the source region for the reported emissions is found to be $L_{\text {Source }}=4.62$. The higher $L$-value of the source region compared to the observation station Jammu ( $L=1.17$ ) shows that the wave may have propagated towards significantly lower latitudes (Smirnova et al., 1976).

Helliwell (1967) was the first to suggest the idea of chorus emission and explained how the frequency spectrum of discrete elements is formed. He further developed a phenomenological model to study the generation of discrete chorus emissions. In this model, the idea of second-order cyclotron resonance of energetic electrons with whistlermode waves first formulated and it explained about the numerous types of discrete emissions. Further analytical and computational calculations (Karpman, 1974; Nunn, 1974) confirmed the idea of the second order cyclotron resonance and permitted that a connection to be made through nonlinear currents, between the parameters of a triggered emission and quasi-monochromatic whistler wave.

Trakhtengerts (1999) suggested a generation mechanism of discrete chorus emissions based on the backward wave oscillator (BWO) regime of magnetospheric cyclotron maser (Trakhtengerts, 1995). Trakhtengerts (1995) revealed that the step like deformation could be the cause of new generation regimes of the cyclotron instability, leading to a succession of discrete chorus signals. Verification of the BWO model of VLF chorus generation using data from MAGION 5 satellite was successfully explained by Titova et al. (2003). Recently, Trakhtengerts et al. (2007) analyzed the Cluster data to show the dependence of the frequency spectrum of individual chorus elements on the position of the observation point in and near the generation region on two different geomagnetically active days using BWO theory. Singh and Singh (2004) have applied BWO theory successfully to explain the generation of discrete VLF emissions observed at low latitudes. Recently, Omura et al. (2008) analyzed the generation process of chorus emissions by both theory and simulation. All the above discussed theories are valid for the rising tone chorus emissions and till now there is not any appropriate theory to account for the falling tone emissions.

\section{Conclusions}

In this paper, we have reported characteristics of VLF chorus emissions recorded at low latitude ground station Jammu. These discrete chorus emissions are falling tones which were rarely observed at any low latitude ground stations. These discrete chorus emissions were generated during a strong geomagnetic storm period of 2-7 May, 1998. The source region of these emissions are between $L=4.5$ to 4.62 . The computed frequency sweep rate of the individual chorus elements varies between -4.25 to $-11.01 \mathrm{kHz} / \mathrm{sec}$ with average repetition period from 0.2 to $0.43 \mathrm{sec}$. It is also observed that the sweep rate increases with time. These results are very interesting to be observed at any low latitude ground station. Further study is required to explain their generation mechanism.

Acknowledgments. The work is partly supported by DST, New Delhi under SERC project and partly by ISRO, Bangalore. R. P. Patel is thankful to DST, New Delhi for awarding research project under SERC-Fast Track Scheme for young Scientists (SR/FTP/PS-12/2006). K. K. Singh is thankful to CSIR for awarding RA. We are thankful to both the reviewers for their valuable comments and suggestions to improve the quality of paper.

\section{References}

Andronov, A. A. and V. Y. Trakhtengerts, Kinetic instability of Earth's outer radiation belt, Geommagn. Aeron., 4, 233-242, 1964.

Bortnik, J., R. M. Thorne, and N. P. Meredith, The unexpected origin of plasmaspheric hiss from discrete chorus emissions, Nature, 452(6), doi:10.1038/nature06741, 2008.

Burtis, W. J. and R. A. Helliwell, Magnetospheric Chorus: Occurance pattern and normalized frequency, Planet. Space Sci., 24, 1007-1024, 1976.

Cornilleau-Wehrlin, N., F. Gendrin, R. Lefeuvre, M. Parrot, R. Garad, and D. Jones, VLF waves observed on GEOS-I, Space Sci. Rev., 22, 371382, 1978.

Hattori, K. and M. Hayakawa, Consideration of dynamic spectra and direction finding results of hiss triggered chorus emissions, Proc. NIPR Symp., Upper Atmosphere Phys., 7, 40-52, 1994.

Hattori, K., M. Hayakawa, D. Lagoutte, M. Parrot, and F. Lefeuvre, Further evidence of triggered chorus emissions from wavelets in the hiss band, Planet. Space Sci., 39, 1465-1472, 1991.

Helliwell, R. A., Whistler and Related Ionospheric Phenomena, Stanford 
Univ. Press, Stanford, USA, 1965.

Helliwell, R. A., A theory of discrete emissions from magnetosphere, $J$. Geophys. Res., 72, 4773-4790, 1967.

Karpman, V. I., Non linear effects in the ELF waves propagating along the magnetic field in magnetosphere, Space Sci. Rev., 16, 361-388, 1974.

Kennel, C. F. and H. E. Petschek, Limit on stably trapped particle fluxes, J. Geophys. Res., 71, 1-28, 1966.

Lauben, D. S., U. S. Inan, T. F. Bell, and D. A. Gurnett, Source characteristic of ELF/VLF chorus, J. Geophys. Res., 107(A12), 1429-1446, 2002.

Nunn, D., A self-consistent theory of triggered VLF emissions, Planet. Space Sci., 22, 349-378, 1974.

Omura, Y., Y. Katoh, and D. Summers, Theory and simulation of the generation of whistler-mode chorus, J. Geophys. Res., 113, A04223, doi:10.1029/2007JA012622, 2008.

Santolik, O., New results of investigations of whistler-mode chorus emissions, Nonlinear Processes Geophys., 15, 621-630, 2008.

Santolik, O. and D. A. Gurnett, Transverse dimensions of chorus in the source region, 30, 1031, 2003.

Sazhin, S. S. and M. Hayakawa, Magnetospheric chorus emissions: a review, Planet. Space Sci., 40, 681-697, 1992.

Singh, A. K. and K. Ronnmark, A generation mechanism for VLF chorus emisions observed at low latitude ground station, Ann. Geophys., 22, 2067-2072, 2004.

Singh, A. K. and R. P. Singh, Observations of discrete VLF emissions at low latitudes and their generation mechanism, Earth Planets Space, 56, 1067-1074, 2004.

Singh, R. P. and R. P. Patel, Hiss-triggered chorus emissions at Indian stations, J. Atmos. Sol.-Terr. Phys., 66, 1027-1033, 2004.

Singh, R., R. P. Patel, R. P. Singh, and Lalmani, An experimental study of hiss triggered chorus emissions at low latitude, Earth Planets Space, 52(1), 37-40, 2000.
Singh, S., R. P. Patel, K. K. Singh, A. K. Singh, and R. P. Singh, Role of geomagnetic disturbances on VLF whistler wave activity at low latitudes, Planet. Space Sci., doi.10.1016/pss-2007.02.001, 2007.

Smirnova, N. A., A fine structure of the ground observed VHF chorus as an indicator of the wave particle interaction process in the magnetosphere, Planet. Space Sci., 32(4), 425-438, 1984.

Smirnova, N. A., P. Yu. Novkov, N. G. Kleimenova, and E. E. Titova, Some spectral peculiarities of VLF emissions registered on the Earth surface near the plasmapause projection, J. Atmos. Terr. Phys., 38, 1247, 1976.

Smith, A. J., M. P. Freeman, and G. D. Reeves, Post midnight VLF chorus events, a substorm signature observed at the ground near $\mathrm{L}=4, J$. Geophys. Res., 101(A11), 24641-24653, 1996.

Titova, E. E., B. V. Kozelov, F. Jiricek, A. G. Demekhov, and V. Yu. Traktengerts, Verification of the backward wave oscillator model of VLF chorus generation using data from MAGION 5 satellite, Ann. Geophys., 21, 1-9, 2003.

Trakhtengerts, V. Y., Magnetosphere cyclotron maser: Backward wave oscillator generation regime, J. Geophys. Res., 100(A9), 17205-17210, 1995.

Trakhtengerts, V. Y., A generation mechanism for chorus emissions, Ann Geophys., 17, 95-100, 1999.

Trakhtengerts, V. Y., A. G. Demekhov, E. E. Titova, B. V. Kozelov, O Santolik, E. Macusova, D. Gurnett, J. S. Pickett, M. J. Rycroft, and D. Nunn, Formation of VLF chorus frequency spectrum: Cluster data and comparison with the backward wave oscillator model, Geophys. Res. Lett., 34, L02104, doi:10.1029/2006 GL027953, 2007.

Tsurutani, B. T. and E. J. Smith, Post midnight chorus: a substorm phenomenon, J. Geophys. Res., 79, 118-127, 1974.

A. K. Singh (e-mail: abhay_s@rediffmail.com), R. P. Patel, R. Singh, K. K. Singh, and A. K. Singh 